\title{
A Simple and Efficient Timing Offset Estimation for OFDM Systems
}

\author{
H. Minn and V. K. Bhargava, Fellow, IEEE \\ Department of Electrical and Computer Engineering \\ University of Victoria \\ Victoria, B.C., Canada V8W 3P6. \\ Email: bhargava@ece.uvic.ca
}

\begin{abstract}
A simple timing offset estimation method for orthogonal frequency division multiplexing (OFDM) systems as a modification to Schmidl \& Cox's method [14] is presented. By designing the training symbol, the timing metric plateau inherent in [14] is eliminated and hence performance is improved. The performances of the proposed method and [14] are evaluated by computer simulation in terms of estimator variance. The timing offset estimator of [16] is also included in the performance comparison as another reference. The simulation results show that the proposed method achieves significantly smaller estimator variance. Using more samples in calculation of half symbol energy required in timing metric is shown to give more robustness against dispersive channel.
\end{abstract}

\section{Introduction}

OFDM has been a major interest for wireline and wireless applications (e.g., ADSL, DAB[1], DVB[2], wireless LAN [3][5], and cellular and PCS data [4]) due to its high data rate transmission capability with high bandwidth efficiency, its robustness to multipath delay spread and its feasibility in applying adaptive modulation and/or power distribution across the subcarriers according to the channel conditions. However, it is also accompanied with many practical issues and one major issue is synchronization. OFDM systems are much more sensitive to synchronization errors than single carrier systems [6], [7]. Synchronization in OFDM usually includes symbol timing synchronization, carrier frequency synchronization and sampling clock synchronization. The first two are more dominant factors and usually performance of frequency synchronization also depends on the accuracy of symbol timing synchronization. Hence, high accuracy of symbol timing synchronization is much desirable and in this paper, only symbol timing synchronization is considered.

Regarding symbol timing synchronization, [8-9] proposes to use the correlation of cyclic prefix with its copy part. [10] also uses correlation of cyclic prefix in a two stage timing estimation but it has long acquisition time. Since guard interval (cyclic prefix) is usually affected by intersymbol interference (ISI), the result of the methods using correlation of cyclic prefix depends on the $a$ priori assumption about the channel. Hence, $[11,12]$ use a larger guard interval where ISI free part of the guard interval is used for timing estimation. However, it fails under some channel conditions and they propose a second method where by making use of the channel estimate, timing metric is maximized by a search varying the position of FFT window. Hence, the acquisition time may not allow

This work was supported by a Strategic Project Grant from the Natural Sciences and Engineering Research Council (NSERC) of Canada. burst mode transmission and the behavior of tracking loop in mobile environment remains subject to further investigation. In [13], an $m$-sequence or chirp synchronization burst is used which is correlated at the receiver with locally generated one. Due to frequency offset, locally generated synchronization burst would be different from the received one. Hence the timing estimation would not be reliable in the presence of large frequency offset. [14] proposes a robust method using a training symbol with two identical halve which can be applied in either continuous or burst mode transmission. However, the timing metric plateau inherent in it causes uncertainty in choosing the best timing point, hence causing large timing estimator variance. In [15] differential in frequency detection together with raising fourth power of the signal to cancel QPSK modulation format is applied. However, initial timing offset is required to be within one-eighth of the FFT size. Alternatively in [16], cyclic prefix and pilot symbols used for channel estimation are exploited for timing estimation.

In brief, most of the previous timing estimation methods have one or more of the following drawbacks: (a) not applicable for both burst mode and continuous mode transmission, (b) depending on the $a$ priori assumption about the channel, (c) restricted to some modulation formats, (d) limited for small frequency offset cases, and (e) having tendency of estimator performance degradation caused by timing metric plateau. In this paper we present a timing offset estimation as a modification to Schmidl \& Cox's method [14]. By eliminating timing metric plateau inherent in [14], the proposed method also avoids the drawbacks described above. The paper is organized as follows. Section II briefly describes the system considered and the timing estimation method of [14]. Section III presents the proposed method. In section IV, the performance of the proposed method and the methods of [14] and [16] are compared in terms of estimator variance obtained by simulation. Conclusions are given in section $\mathrm{V}$.

\section{System Description}

The samples of transmitted baseband OFDM symbol can be given by

$s(k)=\frac{1}{\sqrt{N}} \sum_{n=0}^{N_{u}-1} c_{n} \exp (j 2 \pi k n / N),-N_{g} \leq k \leq N-1$,

where $c_{n}$ is modulated data on the $n^{\text {th }}$ subcarrier, $N$ is the number of inverse Fast Fourier Transform (IFFT) points, $N_{u}(\leq N)$ is the number of subcarri- 
ers, $N_{g}$ is the number of guard samples, $j=\sqrt{-1}$ and the sampling period is $T_{u} / N$ with $1 / T_{u}$ being subcarrier spacing. Consider a discrete-time channel characterized by

$$
h(k)=\sum_{l=0}^{K-1} h_{l} \delta\left(k-\tau_{l}\right)
$$

where $\delta(k)$ represents dirac-delta function, $\left\{h_{l}\right\}$ complex path gains, $\left\{\tau_{l}\right\}$ path time delays which are assumed in multiple of OFDM samples, and $K$ the total number of paths. Then the received samples, assuming perfect sampling clock, can be given by

$$
r(k)=\exp (j 2 \pi k v / N) \sum_{l=0}^{K-1} h_{l} s\left(k-\tau_{l}+\varepsilon\right)+n(k)
$$

where $v$ is the carrier frequency offset normalized to subcarrier spacing, $\varepsilon$ is timing offset in units of OFDM samples and $n(k)$ is the sample of zero mean complex Gaussian noise process with variance $\sigma_{n}^{2}$. Suppose the sample indexes of perfectly synchronized OFDM symbol be $\left\{-N_{g}, \ldots,-1,0,1, \ldots, N-1\right\}$ and maximum channel delay spread be $\tau_{\max }$. Then if $\varepsilon \in\left\{-N_{g}+\tau_{\max },-N_{g}+\tau_{\max }+1, \ldots, 0\right\}$, the orthogonality among the subcarriers will not be destroyed and the timing offset will only introduce a phase rotation in every subcarrier symbol $Y_{m}$ at the FFT output as

$Y_{m}=\exp (j 2 \pi m \varepsilon / N) c_{m} H_{m}+n_{m},-N_{g}+\tau_{\max } \leq \varepsilon<0$

where $m$ is subcarrier index, $H_{m}$ is channel frequency response to the $m^{\text {th }}$ subchannel, i.e., $\left\{H_{m}\right\}=$ $\operatorname{DFT}(h(k))$, and $n_{m}$ is complex Gaussian noise term. For coherent system, this phase rotation is compensated in channel equalization which sees it as a channel introduced phase shift. If the timing estimate is outside the above range, the orthogonality among the subcarriers will be destroyed by the resulting ISI. Then the FFT output subcarrier symbols are given by

$$
\begin{array}{r}
Y_{m}=\exp (j 2 \pi m \varepsilon / N) \frac{N-\varepsilon}{N} c_{m} H_{m}+n_{m}+I_{\varepsilon}(m), \\
\varepsilon<-N_{g}+\tau_{\text {max }} \text { or } \varepsilon>0
\end{array}
$$

where the extra term $I_{\varepsilon}(m)$ results from ISI and interchannel interference (ICI) caused by the loss of orthogonality among the subcarriers (see [13] for details). The useful term also suffers a slight gain loss but for large $N$, it is negiligible. The main detrimental effect is caused by the extra ISI+ICI term. Thus, the guard interval should be long enough for the timing estimate to lie within the range described above. On the other hand, longer guard interval translates into more system capacity loss due to overhead. Hence high performance timing synchronization is much desirable for higher system performance and capacity efficiency. The smaller the variance of timing estimator is, the shorter the guard interval can be designed, and hence less overhead and increased capacity efficiency can be obtained. With this aspect, the performance of the symbol timing synchronization algorithms will be evaluated by the timing estimator variance.

The symbol timing estimator finds the start of the OFDM symbol. The correct symbol timing point will be taken as the start of the useful part of the OFDM symbol (i.e., the start of FFT window for demodulation). Let the training symbol (excluding cyclic prefix) contain two identical halves in time domain each having $L=N / 2$ samples. At the receiver there will be a phase difference between the samples in the first half and their replica in the second half caused by the carrier frequency offset. Training data is usually a PN sequence. Then the Schmidl \& Cox's timing estimator [14] takes as the start of the symbol the maximum point of the timing metric given by

$$
M_{1}(d)=\frac{\left|P_{1}(d)\right|^{2}}{R_{1}^{2}(d)}
$$

where $d$ is a time index corresponding to the first sample in a window of $2 L$ samples and $P_{1}(d)$ is correlation metric given by

$$
P_{1}(d)=\sum_{m=0}^{L-1} r^{*}(d+m) \cdot r(d+m+L)
$$

where ()$^{*}$ represents conjugation and $R_{1}(d)$ represents energy of half OFDM symbol and is included for normalization of the correlation metric in account for the large fluctuation of the OFDM sample amplitudes and given by

$$
R_{1}(d)=\sum_{m=0}^{L-1}|r(d+m+L)|^{2}
$$

The timing metric reaches a plateau (see Fig. 2) which leads to some uncertainty as to the start of the frame. To alleviate this, [14] proposes an averaging method where the maximum point is first found and then two points with $90 \%$ of the maximum value, one to the left and the other to the right of the maximum point, are found. The timing estimate is taken as the average of the two $90 \%$ points.

\section{Proposed Method}

The samples of the training symbol (excluding cyclic prefix) are designed to be of the form

$$
s=\left[\begin{array}{llll}
A & A & -A & -A
\end{array}\right]
$$

where A represents samples of length $L=N / 4$ generated by $N / 4$ point IFF' of $N_{u} / 4$ length modulated data of a PN sequence. If desired, the abrupt amplitude change due to sign conversion in the training symbol can easily be avoided by modifying the PN sequence such that the sum of the corresponding modulated data equals zero. The timing metric is given by

$$
M_{2}(d)=\frac{\left|P_{2}(d)\right|^{2}}{R_{2}^{2}(d)}
$$



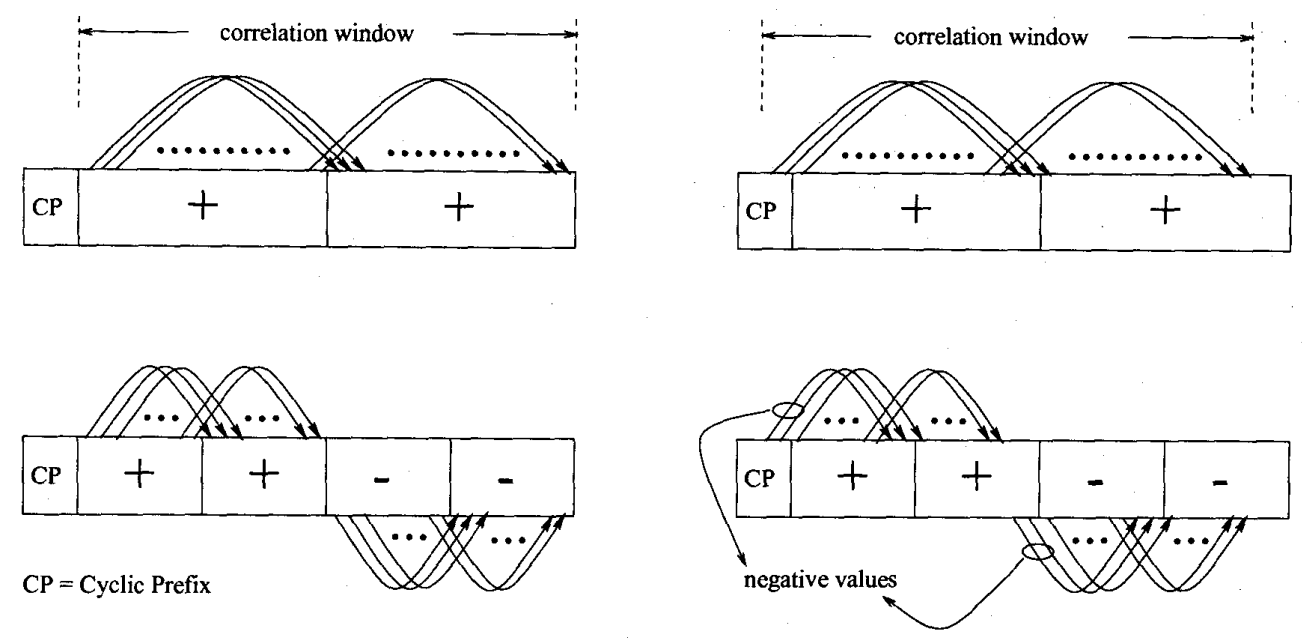

(a) at exact symbol timing point

(b) before exact symbol timing point

Fig. 1. Correlation of the training symbol: Schmidl \& Cox's method (upper), Proposed method (lower)

where

$P_{2}(d)=\sum_{k=0}^{1} \sum_{m=0}^{L-1} r^{*}(d+2 L k+m) \cdot r(d+2 L k+m+L)$

and

$$
R_{2}(d)=\sum_{k=0}^{1} \sum_{m=0}^{L-1}|r(d+2 L k+m+L)|^{2}
$$

In above equations, $P_{2}(d)$ and $R_{2}(d)$ can be calculated iteratively. The training symbol patterns of [14] and proposed method are shown in Fig.(1). Also shown is the elimination of timing metric plateau inherent in [14] by proper design of the training symbol in the proposed method. For training symbol of [14], the correlation metric has its peak for the whole interval of cyclic prefix (under no noise and distortion condition) while the proposed training symbol has its peak of correlation metric at the correct FFT window starting point since off that point, correlation of some samples results in negative values as shown in Fig.(1) hence eliminating the correlation metric peak plateau.

The timing metrics of [14] (Eq. (6)), and the proposed method (Eq. (10)) are shown in Fig. 2 under no noise and distortion condition with the system parameters given in section IV. The correct timing point (index 0 in the figure) is taken as the start of the useful part of training symbol (after cyclic prefix). As discussed previously, the proposed method does not have timing metric plateau and consequently it achieves better accuracy in timing offset estimation. In calculation of half symbol energy, $N$ samples instead of $N / 2$ samples can be used to get more reliable result. Then for both methods, $R_{1}(d)$ and $R_{2}(d)$ can be replaced by the following:

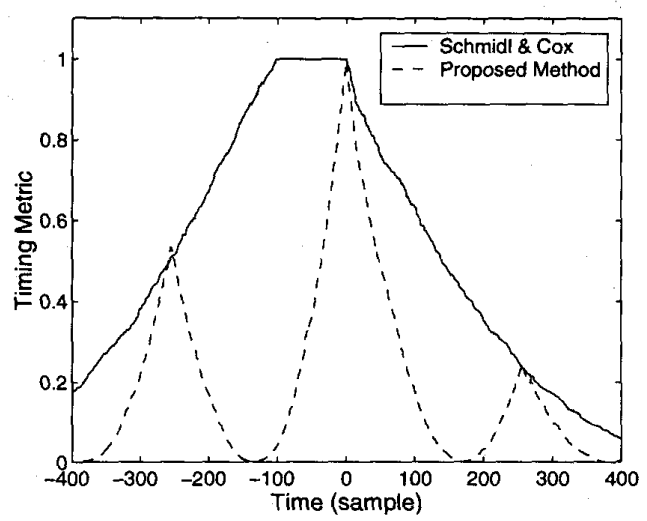

Fig. 2. Timing metric under no noise and distortion condition

$$
R(d)=\frac{1}{2} \sum_{m=0}^{N-1}|r(d+m)|^{2}
$$

\section{Simulation Results and discussion}

Performance of the timing offset estimators have been investigated by computer simulation for five cases: (I) Schmidl \& Cox method (Eq. (6) with $90 \%$ maximum points averaging), (II) Schmidl \& Cox method with modified $R(d)$ (same as (I), except that $R_{1}(d)$ is replaced by $R(d)$ ), (III) the method from [16], (IV) Proposed Method (Eq. (10)), and (V) Proposed Method using $R(d)$ in stead of $R_{2}(d)$. The system used is 1000 subcarriers OFDM system with 1024 point FFT, $10 \%$ guard interval (102 samples), carrier frequency offset of 12.4 subcarrier spacing, QPSK modulation and 10000 simulation runs. Two channel conditions 


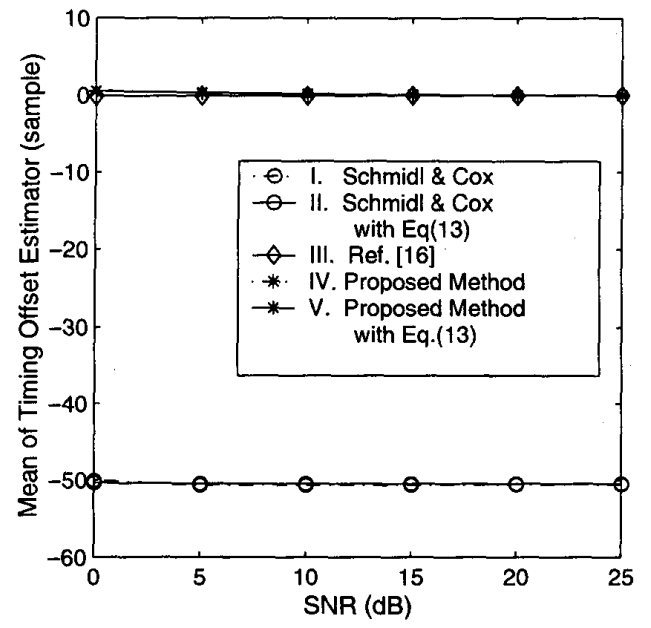

Fig. 3. Mean of the timing offset estimators in AWGN channel

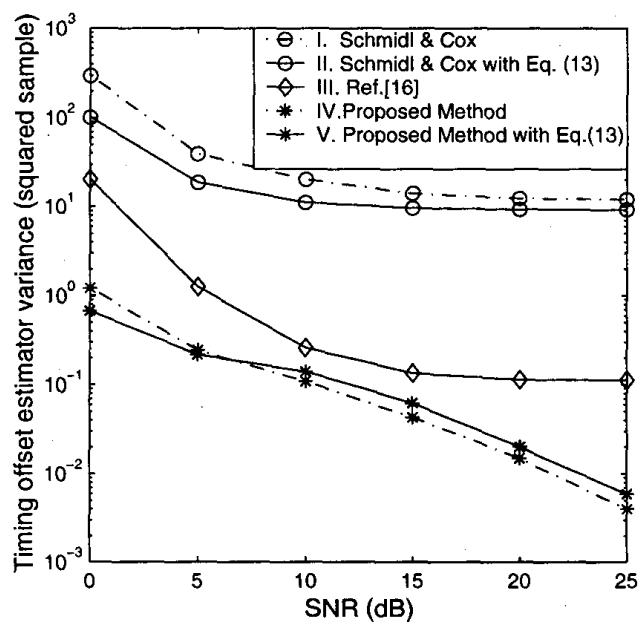

Fig. 4. Timing offset estimator variance in AWGN channel

are considered: a) An AWGN channel with no intersymbol interference (ISI) (it will be called AWGN channel) and b) An AWGN channel with ISI (it will be called ISI channel). The ISI channel is modelled as sixteen paths with path delays $\tau_{i}$ of $0,4,8, \ldots, 60$ samples and path gains given by

$$
h_{i}=\frac{\exp \left(-\tau_{i} / 60\right)}{\sqrt{\sum_{k=1}^{16} \exp \left(-\tau_{k} / 30\right)}}, \quad i=1,2 \ldots, 16 .
$$

For the timing estimator of [16], the additional parameters are: one pilot every $40 t h$ subcarrier and dummy SNR value $S \tilde{N} R=5 d B$.

The means and variances of the timing offset estimates in AWGN channel are shown in Figs. (3) and (4), and in ISI channel are in Figs. (5) and (6) respec-

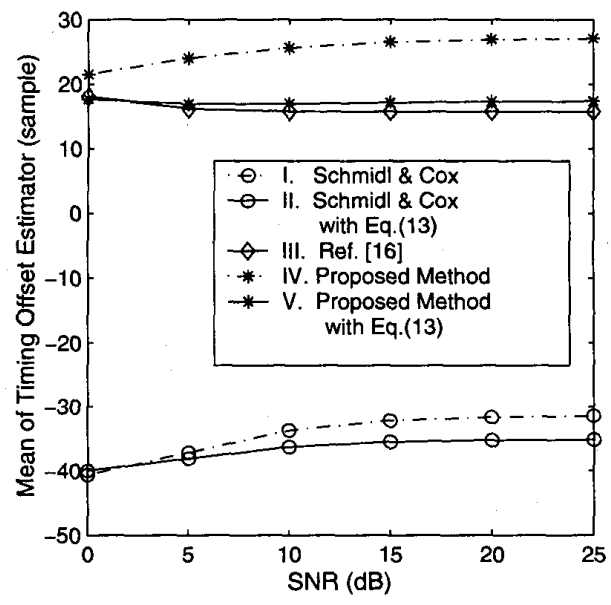

Fig. 5. Mean of the timing offset estimators in ISI channel

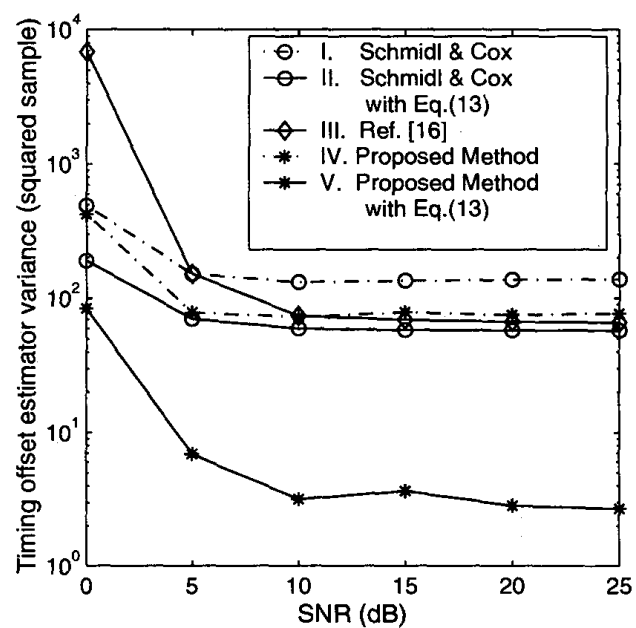

Fig. 6. Timing offset estimator variance in ISI channel

tively. For AWGN channel, the means of the cases I and II are about the middle of timing metric plateau (within the cyclic prefix) while the means of the cases III, IV, and V are about the correct timing point. For ISI channel, the means are observed to be shifted to the right in time axis (i.e. delayed) by some amount depending on the shapes of the timing metric and ISI channel profile. Note that if the timing estimate is desired to lie within guard interval, then the means of the cases III, IV, and V can easily be shifted to the left (i.e., advanced) by appropriate design amount. As for the variances, the following are observed:

- In AWGN channel, cases I, II and III show floor in estimator variance curves while the proposed methods (cases IV and V) do not. In ISI channel, all methods have estimator variance floor.

- Performance of case II is better than case I for all conditions considered, at the expense of some 
additional complexity. The smaller variance of case II is due to using more samples to calculate half symbol energy used in timing metric.

- Proposed methods (cases IV and V) have significantly smaller estimator variance than Schmidl \& Cox's method (case I) for all conditions considered. The better performance of the proposed methods is generally due to the absence of timing metric plateau.

- Comparing proposed methods to case III (Ref.[16]), in AWGN channel, we see that cases IV and $V$ have significantly smaller estimator variance; while in ISI channel, case IV has slightly larger variance for SNR values greater than $10 \mathrm{~dB}$ but case $\mathrm{V}$ has significantly smaller variances.

- In AWGN channel, the slope of the timing metric off the correct timing point determines the estimator variance (see cases I and II vs cases IV, and $\mathrm{V}$ in Fig. 4).

- In ISI channel, using all the samples over one symbol period (excluding cyclic prefix) to calculate the half symbol energy (i.e. using $R$ ) has more effect on reducing the estimator variance than the slope of the timing metric (see case IV vs. cases II and V in Fig. 6).

- As an overall evaluation for both channel conditions, case V (proposed method using $R$ ) performs the best.

The cases II and V have some additional complexity due to using $R$ as compared to the case $I$. However, cases IV and $V$ do not need averaging of timing metric as required in cases I and II. Hence case IV has even smaller complexity than case I.

Similar to the proposed training symbol in this paper which is composed of 4 parts, the training symbol can be designed to be composed of more parts and have steeper roll-off timing metric off the correct timing point so that better timing estimation can be achieved.

\section{Conclusions}

A simple timing offset estimation method for OFDM systems is presented as a modification to Schmidl \& Cox's method [14] where a training symbol containing two identical halves is used. The proposed method uses a training symbol containing four equal length parts: the first two are identical and the last two are the negative of the first two. By eliminating the timing metric plateau inherent in [14], the proposed method achieves a performance improvement. Moreover, using all samples over one symbol period (excluding guard interval), instead of over half symbol period, in the calculation of half symbol energy required in timing metric is shown to give more robustness to dispersive channel. The simulation results show that proposed method has significantly smaller estimator variance than [14] under both AWGN channel and ISI channel. The performance of the method in [16] is also included in the comparison as another reference. As an overall performance for both channels, the proposed method using all samples over one symbol period in calculation of half symbol energy gives the best result.

\section{References}

[1] P. Shelswell, "The COFDM modulation system: the heart of digital audio broadcasting," Elec. Commun. Eng. Journal, June 1995, pp. 127-136.

[2] U. Reimers, "Digital video broadcasting," IEEE Communications Magazine, Vol. 36, No. 6, Jun 1998, pp. 104-110.

[3] M. Aldinger, "Multicarrier COFDM scheme in high bit rate radio local area networks," Proc. PIMRC, 1994, pp. 969973.

[4] L.J. Cimini, Jr., J.C. Chuang and N.R. Sollenberger, "Advanced cellular internet services," IEEE Communications Magazine, Vol. 36, No. 10, Oct 1998, pp. 150-159.

[5] J.C-I. Chuang and N.R. Sollenberger, "Wideband wireless data access based on OFDM and dynamic packet assignment," Wireless Communications and Networking Conf., New Orleans, USA, Sept. 1999, pp. 757-761.

[6] T. Pollet, M. Van Bladel and M. Moeneclaey, "BER sensitivity of OFDM systems to carrier frequency offset and wiener phase noise," IEEE Trans. on Comms., Vol. 43, No. 2/3/4, Feb/Mar/Apr 1995, pp. 191-193.

[7] M. Gudmundson and P.O. Anderson, "Adjacent channel interference in an OFDM system," Proc. Vehicular Tech. Conf, Atlanta, GA, May 1996, pp. 918-922.

[8] J-J. van de Beek, M. Sandell and P.O. Börjesson, "ML estimation of time and frequency offset in OFDM systems" IEEE Trans. Signal Proc., Vol. 45, no. 7, July 1997, pp. 1800-1805.

[9] D. Landström, J.M. Arenas, J.J van de Beek, P.O. Börjesson, M-L.Boucheret and P.Ödling, "Time and frequency offset estimation in OFDM systems employing pulse shaping," Proc. Intl Conf. on Universal Personal Communications, San Diego, CA, USA, Vol. 45, no. 7 , Oct 1997, pp. 279-283.

[10] D. Lee and $K$. Cheun, "A new symbol timing recovery algorithm for OFDM systems," IEEE Trans. on Consum. Electronics, Vol. 43, No. 3, Aug 1997, pp. 767-775.

[11] M. Speth, F. Classen and H. Meyr, "Frame synchronization of OFDM systems in frequency selective fading channels," Proc. Vehicular Tech. Conf., Phoenix, Arizona, USA, May 1997, pp. 1807-1811.

[12] M. Speth, D. Daecke and H. Meyr, "Minimum overhead burst synchronization for OFDM based broadband transmission," Proc. Global Telecom. Conf., Sydney, Australia, Nov 1998, pp. 2777-2782.

[13] L. Hazy and M. El-Tanany, "Synchronization of OFDM systems over frequency selective fading channels," Proc. Vehicular Tech. Conf., Phoenix, Arizona, USA, May 1997, pp. 2094-2098.

(14] T.M. Schmidl and D.C. Cox, "Robust frequency and timing synchronization for OFDM," IEEE Trans. on Comms., Vol. 45, No. 12, Dec 1997, pp. 1613-1621.

[15] B. McNair, L.J. Cimini Jr. and N. Sollenberger, "A robust timing and frequency offset estimation scheme for orthogonal frequency division multiplexing (OFDM) systems," Proc. Vehicular Tech. Conf., Houston, Texas, USA, May 1999, pp. 690-694.

[16] D. Landström, S.K. Wilson, J.J. van de Beek, P. Ödling and P.O. Börjesson, "Symbol time offset estimation in coherent OFDM systems," Proc. Intl Conf. on Communications, Vancouver, BC, Canada, June 1999, pp. 500-505. 